\title{
Évaluation des pratiques de gestion de la diversité du niébé (Vigna unguiculata) : une tentative méthodologique expérimentée au Bénin
}

\author{
Mohamed Nasser Baco ${ }^{1,5}$ \\ Adam Ahanchedé ${ }^{2}$ \\ Saliou Bello ${ }^{1}$ \\ Alexandre Dansi $i^{3}$ \\ Raymond Vodouhè ${ }^{4}$ \\ Gauthier Biaou ${ }^{2}$ \\ Jean-Paul Lescure ${ }^{5}$ \\ 1 Institut national des recherches agricoles \\ du Bénin (Inrab), \\ BP 27, \\ Parakou \\ <bello_saliou@yahoo.fr> \\ ${ }^{2}$ Faculté des sciences agronomiques \\ du Bénin, \\ Université d'Abomey Calavi, \\ 01 BP 526 \\ Cotonou \\ Bénin \\ $<$ <hanchedeadam@yahoo.fr> \\ <yahoo.frgbiaou@yahoo.fr> \\ ${ }^{3}$ Faculté des sciences et techniques, \\ Université d'Abomey Calavi, \\ 01 BP 4521 \\ Cotonou \\ Bénin \\ <adansi2001@yahoo.fr> \\ ${ }^{4}$ Bioversity International, \\ West and Central Africa, \\ 08 BP 0932 Tri Postal, \\ Cotonou \\ Bénin \\ <r.vodouhe@cgiar.org> \\ ${ }^{5}$ Institut de recherche pour le \\ développement (IRD), \\ Unité de recherche (UR) 168 " Dynamiques \\ environnementales entre forêt, agriculture \\ et biodiversité: des pratiques locales \\ sur la nature aux politiques publiques "., \\ Centre IRD d'Orléans, \\ 5 rue du carbone, \\ 45072 Orléans cedex 2 \\ $<$ nasser.baco@orleans.ird.fr> \\ $<$ jean-paul.lescure@orleans.ird.fr>
}

Tirés à part : M.N. Baco

\begin{abstract}
Résumé
Depuis la convention sur la biodiversité, l'évaluation de la richesse variétale comme celle des pratiques qui lui sont associées, est devenue un préalable à toute tentative de conservation in situ. La méthode participative que nous avons expérimentée au Bénin dans le village de Sori sur la culture du niébé (Vigna unguiculata L.), tente de répondre à cette attente. Cette étude a permis de recenser quatorze variétés nommées par les paysans à Sori. Dix méga-pratiques contribuant à maintenir ou à renforcer le pool variétal ont été inventoriées et décrites. Leur contribution aux objectifs de maintien de la diversité des usages, d'optimisation des ressources de l'exploitation et de stratégies antirisques a été analysée. Pour chaque pratique, une valeur d'aptitude à la conservation a été calculée. Des valeurs élevées ont été obtenues pour les pratiques culturales telles que la préservation des récoltes par les insecticides végétaux, des techniques de multiplication des semences ou encore des associations culturales. L'étude a aussi montré comment les variétés locales sont incorporées dans les systèmes de production et/ou les politiques agricoles et les stratégies de conservation de la biodiversité.
\end{abstract}

Mots clés : agrobiodiversité ; Bénin ; conservation des ressources ; critère de sélection ; niébé.

Thèmes : productions végétales ; ressources naturelles et environnement.

\section{Abstract}

Evaluation of cowpea (Vigna unguiculata) diversity management practices: A methodological approach tested in the village of Sori (Benin, West Africa)

Since the Convention on Biological Diversity, the evaluation of varietal richness and its inherent management practices have become prerequisite tasks for in situ conservation. The participative method we are implementing in Sori village (Benin) on cowpea (Vigna unguiculata $\mathrm{L}$ ) is consistant with such expectations. Basing the estimation of landrace richness on their local names, the participative approach used in this study made it possible to inventory 14 landraces in Sori. Ten mega-practices contributing to maintaining and/or enhancing the pool of landraces were inventoried, described and evaluated according to criteria such as the diversity of use, the optimization of farm resources and anti-risk strategies. For each practice, an aptitude value for conservation was calculated. These approaches revealed that botanic treatments, seed multiplication and cultural association are the most important practices according to value markers of agricultural practices. This study made it possible to test the use of particular a methodology in evaluating peasant management practices of agrobiodiversity. It also highlighted how local varieties are taken into account in the production systems and the agricultural policies of biodiversity conservation.

Key words: agrobiodiversity; Benin; cowpeas; resource conservation; selection criteria. Subjects: natural resources and environment; vegetal productions. 
a diversité agricole est confrontée à un environnement changeant gouverné par le marché et on se demande si les pratiques séculaires peuvent toujours permettre de la conserver. En guise de solution, longtemps jugées inaptes à satisfaire les besoins alimentaires et agricoles croissants des populations, les pratiques paysannes de gestion de l'agrobiodiversité sont de plus en plus reconnues pour la conservation in situ (Louette et Smale, 1996), et l'article 10 de la Convention sur la biodiversité, implique que soient identifiées les meilleures pratiques locales susceptibles de maintenir l'agrobiodiversité.

$\mathrm{Au}$ Bénin, et particulièrement au Nord Bénin, avec une population en pleine croissance (taux d'accroissement supérieur à $4 \%$ ), une paupérisation grandissante des ruraux (Banque Mondiale, 2003), la situation de l'agriculture et de sa diversité paraît encore plus critique. Les terres se dégradent et le coton livre une concurrence aux cultures vivrières (Akker van den, 1999). Dans ces conditions, le raccourcissement progressif de la jachère et les techniques culturales épuisant le sol à long terme entraînent une baisse considérable des rendements agricoles et l'abandon de certaines variétés (Zoundjihékpon et al., 1997).

Le niébé (Vigna unguiculata) fait partie des cultures confrontées à cette situation. Qualifié de viande de pauvres (Alzouma, 1995), le niébé est la légumineuse à graine préférée pour ses nombreux usages dans l'alimentation humaine. Le niébé contribue également à la culture du coton en participant à la satisfaction des besoins de subsistance alimentaire d'une importante main-d'œuvre familiale et/ou salariée pendant son installation et sa récolte. Par ailleurs, le niébé permet d'assurer des rotations culturales et se pratique en association avec diverses céréales (maïs, sorgho et mil) pour une gestion rationnelle de la fertilité. Ses fanes assurent également un complément alimentaire pour les ruminants en saison sèche.

Cependant, les superficies de niébé s'amenuisent ainsi que l'intérêt des hommes pour cette culture qui est aujourd'hui majoritairement assurée par les femmes. De plus, l'approvisionnement en semences de niébé reste difficile du fait de l'inorganisation de la filière semencière. Cette situation fait craindre une baisse de sa diversité. Si la nécessité de promouvoir la conservation des variétés locales du niébé devient alors un enjeu, il convient d'inventorier et d'évaluer les pratiques paysannes les plus intéressantes pour maintenir et conserver de manière dynamique cette biodiversité.

L'étude a été conduite dans le village de Sori (Nord Bénin) à l'aide d'un groupement informel de femmes productrices de niébé. De plus, un projet de développement de la culture du niébé a été mis en œuvre dans ce village au début des années 1990, ce qui a favorisé l'introduction de nouvelles variétés et de nouvelles pratiques sur le niébé. Les objectifs de cette étude étaient d'identifier les variétés présentes dans les agrosystèmes de Sori ainsi que les processus et pratiques utilisés pour maintenir cette diversité. Puis, en utilisant une méthode participative, nous avons évalué ces pratiques pour identifier celles qui pourraient être intégrées dans la politique agricole de conservation de la diversité du niébé.

\section{Méthodologie}

Le village de Sori est situé à $10,43^{\circ}$ de latitude Nord et $2,46^{\circ}$ de longitude Est. La pluviométrie de la zone varie de 900 à $1000 \mathrm{~mm}$ par an. Le climat va du type soudanien au type soudano-sahélien dans la zone d'étude, caractérisée par une saison sèche de novembre à mai et une saison des pluies de juin à octobre. Sori, situé sur la route interÉtat CotonouNiamey, englobe une soixantaine d'exploitations agricoles et offre au chercheur plusieurs personnes-ressources (encadreurs agricoles, membres d'organisations de paysans, sages du village...). Les personnes enquêtées ont été choisies de manière à constituer un échantillon représentatif du village en tenant compte des ethnies, des classes d'âges et du genre. L'approche suivie se décompose en cinq étapes combinant entretiens particuliers et travail de groupe avec les paysans.

On cherche à créer un premier indice qui valorise les pratiques spécifiquement appliquées aux variétés rares. Pour ce faire, il faut déterminer quelles sont les variétés les plus rares.

\section{Identification et classement des variétés}

La liste des (v) variétés du village est établie à partir d'entretiens individuels et de travail de groupe. Des séances de travail ont été organisées en groupe mixte de producteurs/productrices, de jeunes/ vieux pour dégager les synonymies et éviter les biais liés à la dénomination paysanne des variétés. Ces séances ont été facilitées par la présentation d'au moins un échantillon de graine de chaque variété par les paysans détenteurs.

Pour chaque variété inventoriée des informations sont recueillies sur les superficies allouées (grandes ou petites) et sur le nombre d'exploitations où elle se retrouve (grand ou petit). En fonction des délibérations du groupe de travail, les variétés sont ainsi classées dans une figure appelée "quatre carrés" (Grum et al., 2003).

La classification paysanne considère les superficies allouées à une variété et inférieures à "une corde" (Win en bariba, équivalent à 0,25 hectare) comme petites. Les variétés absentes chez plus de la moitié des paysans procédant à la classification sont considérées comme produites par peu de personnes. L'accord sur la classification de certaines variétés est très difficile à avoir. Dans ces cas, il a fallu procéder à des votes pour départager les paysans.

Les variétés sont aussi classées par le chercheur dans le graphe des quatre carrés, sur la base des médianes, calculées à partir de données variétales recueillies individuellement chez les paysans. Les valeurs inférieures à la médiane sont considérées comme petites et les valeurs supérieures à celle-ci sont considérées comme grandes. Les résultats obtenus sont quasiment les mêmes que ceux obtenus par le classement des paysans. Ils permettent de distinguer les variétés potentiellement menacées des variétés abondantes. La classification sur la base des médianes a finalement été retenue pour la suite de l'analyse afin de permettre les comparaisons à d'autres résultats et les généralisations des conclusions. Mais la classification paysanne, quant à elle, a permis d'impliquer les paysans dans la démarche et de leur faire prendre conscience des menaces de disparition qui existent sur certaines variétés cultivées dans leur village. Les variétés potentiellement menacées (petites surfaces et peu de cultivateurs) se retrouvent rangées dans le carré inférieur gauche (figure 1). Un rang est attribué à chaque variété selon le carré où elle se trouve :

- rang 1, grandes surfaces et nombreuses exploitations ;

-4 petites surfaces et peu d'exploitations ; 


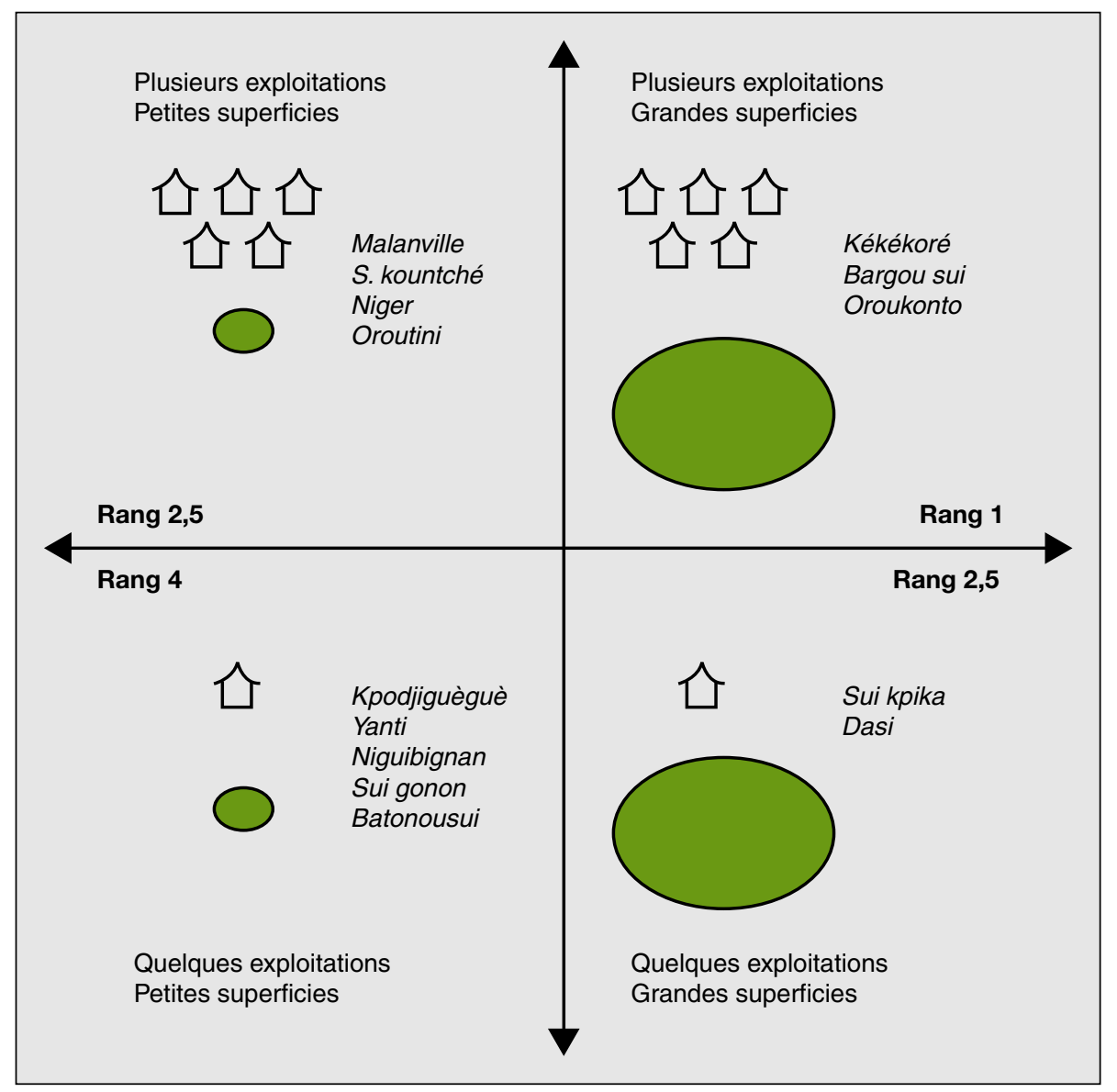

Figure 1. Distribution des variétés de niébé à Sori dans les quatre carrés.

Figure 1. Distribution of Sori cowpea landraces in the four squares.

- rang 2,5 pour les deux autres carrés. Ce rang valorise les variétés les plus menacées.

\section{Identification et classement des pratiques}

On définit comme bonne pratique de conservation des variétés locales tout système, organisation ou processus permettant dans un espace donné (du niveau local au niveau national) de maintenir, d'améliorer ou de créer les ressources génétiques agricoles et d'assurer leur disponibilité aux agriculteurs et autres utilisateurs afin d'améliorer le niveau de vie de façon durable (Grum et al., 2003).

L'inventaire des $(p)$ pratiques associées aux variétés est dressé par le groupe de travail avant de procéder à l'examen individuel des liens existants entre chacune d'elles et chacune des $v$ variétés répertoriées. Les scores, égaux à 0,1 et 2 , sont attribués lorsque les degrés de liaison de la pratique $p$ à la variété sont respective-

$$
\operatorname{MPC} 1=\left(\sum_{1}^{v} \frac{\text { rang } \times \text { score }_{v} \times 100}{8}\right) / v
$$

L'indicateur MPC 1 est élevé pour les pratiques fortement appliquées aux variétés rares.

Calcul d'un second type de valeurs des " marqueurs de pratiques culturales " (MPC 2)

L'objectif de ce second calcul est de prendre en compte, dans l'appréciation des pratiques, les fonctions auxquelles sont destinées les variétés qui leur sont liées. Toujours en groupe, les paysans sont alors conviés à s'exprimer sur l'adéquation de chaque pratique à différents objectifs appelés critères. Trois critères paysans ont été discutés et choisis avec les paysans :

- un grand portefeuille de variétés autorise une grande diversité d'usages. Le critère est nommé "diversifier les usages":

- un grand portefeuille de variétés permet d'optimiser l'utilisation des moyens de production (terre, travail, capital). Le critère est nommé "optimisation des ressources" ;

- un grand portefeuille de variétés permet de faire face au risque. Le critère est nommé "stratégies antirisques ".

Le rôle que chaque pratique joue pour chaque critère s'évalue ensuite à travers une méthode référendaire. On note le suffrage obtenu par chaque pratique à la question: "Cette pratique est-elle bonne pour atteindre les objectifs de ce critère?" Le score obtenu est noté en pourcentage de votes positifs. Le MPC 2 de chaque pratique se calcule ensuite en faisant la moyenne des scores obtenus pour l'ensemble des critères. Sa plage de valeurs va de 0 à 100.

\section{Confrontation des indices}

Un dernier tableau permet de confronter les indices. Les pratiques jugées les plus liées à la conservation variétale sont celles présentant la meilleure moyenne entre MPC 1 et MPC 2. Elles constituent les meilleurs marqueurs de pratiques culturales associées aux variétés rares et permettant de répondre aux objectifs des paysans.

\section{Résultats}

\section{Richesse variétale du niébé}

Quatorze variétés ont été inventoriées à Sori (figure 1) et réparties en quatre 
carrés. Kékékoré, Bargou sui et Oroukonto apparaissent comme étant les variétés les plus cultivées car elles se retrouvent dans le quadrant correspondant à plusieurs exploitations et à grandes superficies. En revanche, cinq variétés (Yanti, Kpodjiguèguè, Sui gonna, Niguibian et Batonousui) semblent avoir une production marginale car très peu d'exploitations les cultivent et sur de petites superficies. Les six autres variétés se distribuent entre les carrés intermédiaires. L'analyse fine du tableau 1 montre qu'il y a aussi bien des variétés provenant du Niger et du Nigeria, que des variétés introduites à partir du sud et d'autres localités du nord du Bénin dans les années 1980 et 1990. Seule la variété Orou tini portant parfois le nom Carder (Centre d'action régional pour le développement rural) est reconnue par les paysans comme provenant des services d'encadrement. Pourtant durant ces vingt dernières années, une demi-dizaine de variétés (Vita 4 et Vita 5, TVX 1850-01F, TVX 3236, IT 82 E 32...) ont été diffusées (Detongnon et Affokpon, 2001).

\section{Les dix pratiques de gestion du niébé}

Dix pratiques contribuant à maintenir ou renforcer le pool variétal du niébé à Sori ont été inventoriées, évaluées et classées par les paysans (tableau 2). Les pratiques associations culturales, cérémonies cultuelles et multiplications des semences sont les plus liées aux variétés rares.

\section{Discussion}

\section{Une diversité variétale du niébé à risque}

En s'intéressant à l'origine et à la période d'introduction, il apparaît que Sori n'est pas le lieu d'origine des variétés Yanti, Kpodjiguèguè, Sui gonan, introduites depuis 1990. Ainsi, la faible production de ces trois variétés serait liée au fait qu'elles ne sont pas dans leur zone traditionnelle de culture. Elle pourrait être liée au système formel de diffusion semencière peu opérationnel dans le Nord. En revanche, les périodes d'introduction de Niguibian et Batonou sui échappent à la mémoire collective. Elles peuvent de ce fait être considérées comme des "variétés locales " dont la production marginale

Tableau 1. Origine et année d'introduction des variétés à Sori.

Table 1. Origin and introduction period of cowpea landraces in the village of Sori.

\begin{tabular}{lccc}
\hline Variétés & Origine & $\begin{array}{c}\text { Niveau } \\
\text { de production }\end{array}$ & $\begin{array}{c}\text { Période } \\
\text { d'introduction }\end{array}$ \\
\hline Seyni kountché & Niger & Moyen & Entre 1980 et 1990 \\
Bargou sui & Nord & Élevé & \\
Kékékoré & Nord & Élevé & \\
Orou tini & Carder & Moyen & \\
Dasi & Nord & Moyen & \\
Oroukonto & Nord & Élevé & Après 1990 \\
Malanville & Nord & Moyen & \\
Niger & Niger & Moyen & \\
Kpodjiguèguè & Sud & Faible & \\
Yanti & Coopérant blanc & Faible & \\
Sui kpika & Nord & Moyen & Inconnue \\
Sui gonan & Nigeria & Faible & \\
Niguibignan & Locale & Faible & \\
Batonousui & Locale & Faible & \\
\hline
\end{tabular}

\section{Tableau 2. Classement des pratiques de gestion du niébé à partir de MPC 1 et MPC 2.}

Table 2. Classification of management practices of cowpea landrace through agricultural marker index value MPC 1 and MPC 2.

\begin{tabular}{lcccc}
\hline \multicolumn{1}{c}{ Pratique } & MPC 1 & MPC 2 & Moyenne & Rang \\
\hline Insecticides botaniques & 29,5 & 100,0 & 64,7 & 1 \\
Multiplication semences & 10,7 & 100,0 & 55,4 & 2 \\
Association culturale & 12,5 & 91,7 & 52,1 & 3 \\
Stockage/grenier & 14,3 & 66,7 & 40,5 & 4 \\
Séchage solaire & 13,4 & 66,7 & 40,0 & 5 \\
Pratique culinaire & 7,1 & 70,0 & 38,6 & 6 \\
Culture de contre-saison & 7,1 & 63,3 & 35,2 & 7 \\
Commercialisation & 10,7 & 50,0 & 30,4 & 8 \\
Cérémonies cultuelles & 5,4 & 50,0 & 27,7 & 9 \\
Fanes pour bétail & 7,1 & 40,0 & 23,6 & 10 \\
\hline \hline
\end{tabular}

concourt à les exposer aux risques de disparition.

\section{Analyse des principales pratiques}

Multiplication des semences

La multiplication des semences dans les champs de case ou dans les jardins conduit à conserver une partie de la production pour un usage semencier. Les variétés telles que Yanti, Sui kpika, Malanville, Kékékoré, Oroukonto demeurent les plus concernées par cette pratique qui demande des précautions que tous les paysans ne maîtrisent pas. En général, lorsqu'une nouvelle variété est introduite dans le champ, elle se cultive d'abord en champ de case ou sur une petite portion de terre minutieusement choisie.

\section{Pratiques culturales}

Assolement rotation et association culturale

Le niébé est intégré dans la rotation des cultures en vue de maintenir la fertilité des sols, d'une part, et d'assurer de meilleurs rendements pour les cultures associées, d'autre part (Carsky et al., 2003). Ce double rôle qu'il joue dans les systèmes de culture constitue un des facteurs importants de la préservation de ses variétés

Oyewole et al. (2000) ont observé que les paysans préfèrent la succession niébé/ maïs à mucuna/maïs, bien que l'arrièreeffet du niébé pour le maïs soit moins bon 
que celui du mucuna. Selon Schulz et al. (2003), le soja et le niébé ont été utilisés pour réduire la densité des graines de Striga hermonthica dans le sol.

Sur le plan des interrelations entre la nutrition en phosphore, en azote et la fixation symbiotique, Sanginga et al. (2000) puis Carsky et al. (2001) ont fait observer que le bilan apparent de l'azote chez le niébé est bonifié par une amélioration de la nodulation grâce à une nutrition suffisante en phosphore, condition nécessaire pour que la symbiose fixatrice d'azote puisse profiter au système solplante.

La pratique d'association culturale englobe les différentes formes d'associations culturales avec le niébé et les logiques qui les guident. Les associations les plus courantes sont celles avec l'igname et les céréales. Dans l'association avec les céréales, les types biologiques cohabitent en symbiose et ce système permet de garder dans les champs les variétés à cycle long de trois à quatre mois. La vente au marché devient aujourd'hui la principale raison de production du niébé par les paysans qui, pour maximiser le profit, préfèrent produire en culture pure des variétés précoces permettant d'assurer deux récoltes par saison de pluies. Dans cette dynamique de redéfinition des objectifs de production vers le marché, les variétés à cycle tardif (Batonou sui et Niguibian...) sont de plus en plus abandonnées et ne sont maintenues dans les systèmes de culture qu'à travers des systèmes d'associations permettant d'optimiser la productivité de la main-d'œuvre.

\section{Culture de contre-saison}

Cette pratique concerne surtout les variétés précoces ( 40 à 60 jours) utilisées par les femmes pendant la saison sèche dans les jardins de case, qui assurent ainsi leur maintien constant et leur évolution au cours du temps. Les variétés Sui Kpika, Sui gonan, Orou Tini, Malanville et Yanti sont les plus concernées par cette pratique.

\section{Pratiques d'usage de la récolte}

\section{Préparations culinaires}

Les formes de consommation du niébé varient en fonction de la variété. Les préparations culinaires permettent de maintenir dans l'agrosystème des variétés à travers leur utilisation et leur facilité d'utilisation culinaires. Les formes de préparation les plus courantes sont les sauces (à base de feuilles et de graines), les beignets (Kolo, Alèlè, Toumbani), le "Waké" (mélange niébé et riz). Les variétés de couleur blanche (Bargou sui,
Kekekorè, Orou Konto, Orou tini, Dasi, Yanti, Sui Kpika, Malanville, Seynikountché) restent les préférées à Sori pour ces différents types de préparations culinaires.

\section{Cérémonies cultuelles}

Les cérémonies rituelles représentent des pratiques sociales animistes assurant une communion avec les ancêtres et les dieux. Les cérémonies d'offrandes aux ancêtres et aux fétiches constituaient un élément important dans la vie culturelle et religieuse des populations traditionnelles béninoises (Tall, 1995). Le niébé fait partie des principales offrandes lors des cérémonies aux divinités protectrices. Par exemple, pour espérer une bonne chasse, des offrandes de mets à base de niébé se font aux esprits et aux ancêtres en début de la saison sèche. Des pratiques similaires sont courantes pour souhaiter la bienvenue aux jumeaux dans une famille et en vue d'une bonne récolte. Les variétés rouges de niébé (Kpodjiguégué...) peu cultivées paraissent les plus utilisées dans ces pratiques, entraînant certains paysans à leur réserver de petites parcelles dans leurs champs.

\section{Commercialisation}

Cette pratique concerne la vente du niébé et la contribution des variétés aux revenus des paysans et des femmes. Elle est l'œuvre des femmes revendeuses qui achètent les graines de niébé à différents endroits après les récoltes et les stockent dans des grands sacs ou dans des tonneaux. Afin de bénéficier d'une meilleure rente commerciale, les femmes ont recours à des traitements pour conserver pendant quelques mois toutes les variétés à commercialiser. L'aptitude d'une variété à être commercialisée regroupe ses caractéristiques organoleptiques, les types de préparations culinaires et son aptitude à être bien conservée avec des produits phytosanitaires accessibles. La variété Kékékoré se conserve mieux avec ces produits que les autres variétés destinées à la commercialisation, ce qui lui confère un avantage comparatif au niveau du prix de vente. Son prix à la commercialisation est une fois et demie plus élevé que celui des autres variétés. Malanville, Seyni kountche, Dasi, Sui kpika et Niger sont les variétés qui s'achètent aussi à des prix compétitifs sur le marché.

\section{Fanes pour bétail}

Cette pratique consiste à valoriser la biomasse du niébé en utilisant les fanes pour nourrir les animaux ou en constituant des bottes de fanes vendues ensuite aux éle- veurs transhumants. Les contrats non monétaires de parcage qui existaient entre éleveurs transhumants et agriculteurs permettant la vaine pâture disparaissent ainsi au profit de nouveaux rapports marchands. Contrairement à la pratique de commercialisation, les hommes sont les principaux acteurs de cette pratique qui s'applique aux 14 variétés de niébé recensées dans cette étude. Les paysans reconnaissent que les variétés comme Batonou sui, Sui Kpika sont celles qui produisent le plus de biomasse.

\section{Pratiques de conservation}

\section{Traitements phytosanitaires naturels} (traitements botaniques)

Il s'agit en fait de traitements postrécolte pour la conservation des graines du niébé. Cette pratique permet aux producteurs de faire face à la non-disponibilité sur le marché des produits de traitement du niébé soumis à une forte pression parasitaire, en utilisant des extraits (liquide, poudre, cendre...) de plantes. Parmi les produits botaniques de conservation utilisés, se trouvent la poudre ou l'huile de neem (Azadiratcha indica), la cendre de karité (Vittellaria paradoxa), la poudre d'écorce de Cailcédrat (Caya senegalensis). La pratique "insecticides botaniques " s'applique aux variétés sensibles aux parasites pendant le stockage: Bargou sui, Kekekoré, Orou Konto, Malanville.

Par ailleurs, l'eau résiduelle de préparation de beurre de karité s'utilise pour combattre les xylophages attaquant les matériaux entrant dans la construction des greniers.

Au nombre des problèmes défavorisant les cultures du niébé par rapport aux céréales, on cite sa faible productivité et sa difficulté de conservation. Certains attribuent d'ailleurs à ces facteurs la tendance des sociétés rurales à limiter la production de légumineuses au fur et à mesure de leur développement (Carsky et al., 2003).

De plus, le parasitisme du niébé est très important en raison de la diversité des nuisibles qui attaquent la culture et de l'échelonnement des dégâts du début de la végétation à la récolte. Les parasites les plus connus sont :

- les nématodes, les chenilles des pousses en début de la végétation ;

- les nombreux insectes comme les punaises coréides, les cicadelles jassides, les thrips, les chenilles de lépidoptères à la floraison ;

- le Prezotrachelus varum, les jassides et les Bemisia sp qui sont des apions au 
cours de la fructification et en fin de cycle ;

- et le Callosobruchus chinensis, un Bruchidae des stocks.

\section{Séchage solaire}

Le séchage solaire consiste à étaler au soleil les semences, soit en gousse soit décortiquées, avant de les conserver, afin de réduire la teneur en eau de manière à éviter les prégerminations et à permettre une bonne levée à la plantation et une bonne viabilité des plantules. Les variétés précoces (Sui kpika, Yanti, Malanville, Sui gonan...) sont les plus soumises à cette pratique.

\section{Stockage/grenier}

Il s'agit de la protection des stocks contre les déprédateurs, en utilisant un produit chimique (Sofagrain, par exemple) mais également en apportant des soins aux greniers ou aux récipients (calebasse, tonneaux, gourdes, jarres, bouteilles) dans lequel les semences se conservent.

\section{Marqueurs de pratiques culturales} et meilleures pratiques

Le MPC 1 est élevé pour les pratiques associées à la culture des variétés fragiles cultivées par quelques rares ménages sur de petites superficies. Il est faible pour les pratiques appliquées surtout aux variétés que l'on retrouve chez la majorité des producteurs et sur de grandes superficies. A travers cet indice, les pesticides botaniques constituent la meilleure pratique avec une valeur d'indice quasiment double de celle des autres. Le MPC 2 confirme cette première place et révèle l'importance des pratiques telles que la multiplication des semences et l'association culturale dans la gestion de la diversité du niébé.

Ce résultat met en lumière les principales difficultés qu'éprouvent les paysans dans le maintien d'une diversité variétale du niébé et qui se résument à la forte pression parasitaire, qui s'exerce plus particulièrement sur certaines de ces variétés et aux difficultés à se procurer les semences certifiées.

Les fanes pour bétail représentent la pratique ayant la valeur des marqueurs de pratiques culturales la plus faible, ce qui pourrait s'expliquer par le fait que les biomasses de toutes les variétés sont concernées par cette pratique. En effet, pour les paysans, les pratiques générali- sées non spécifiques aux variétés et connues de tout le monde n'ont pas de grande portée en matière de gestion de la diversité.

\section{Conclusion}

Conçue pour évaluer les pratiques de gestion variétale, la démarche expérimentée met l'accent sur la contribution de systèmes de gestion traditionnels dans la conservation de l'agrobiodiversité et la valorisation de systèmes agricoles durables.

L'étude a permis de dégager une diversité de variétés insoupçonnée dans la culture du niébé. Les 35 \% des variétés gérées par quelques producteurs sur de petites superficies, dont les variétés Niguibian et Batonousui, semblent les plus concernés par les menaces de disparition dans l'agrosystème de Sori. Il y a néanmoins un système dynamique de gestion paysanne qui tente d'assurer le maintien de ces variétés à partir de pratiques telles que les traitements botaniques, la multiplication de semences et l'association culturale.

En somme, les pratiques de gestion du niébé à Sori sont diverses et multiformes. Cependant, les pratiques d'ordre communautaire (groupes d'entraide, rituelle pour célébrer les nouvelles récoltes...) disparaissent probablement en raison de l'émergence des nouvelles religions et à l'effritement de la cohésion et de la solidarité au sein des paysans. Des efforts sont nécessaires de la part des pouvoirs publics pour assurer la durabilité des pratiques de gestion de l'agrobiodiversité. Il s'agira par exemple de la mise en ouvre de programmes d'amélioration génétique participative, de sélection variétale participation et de l'institutionnalisation de "diversity awards". Il faudra aussi encourager le maintien des usages du niébé, voire les diversifier.

\section{Références}

Akker van den E. Majors crops and their regional distribution in Benin. In : Herrmann L, Ven-
nemann K, Stahr K, Oppen von M, eds. Atlas of natural and agronomic resources of Niger and Benin. Hohenheim : université de Hohenheim, 1999. www.uni-hohenheim.de/atlas308.
Alzouma I. Connaissance et contrôle des coléoptères Bruchidae ravageurs des légumineuses alimentaires au Sahel. Sahel IPM 1995 ; 1 : 2-16.

Banque Mondiale. Benin : poverty reduction strategy paper and joint staff assessment Report 25475-BEN. Washington (DC) : International Development Association (IDA); International Monetary Fund (IMF), 2003.

Carsky RJ, Douthwaite B, Manyong VM, et al. Lessons for appropriate soil management technology generation for the savannas and their application to the grain legume cereal rotation system. Cah Agric 2003 ; 12 : 227-33.

Carsky RJ, Singh BB, Oyewole B. Contribution of early season cowpea to late season maize in the savanna zone of West Africa. Biol Agric Hortic 2001 ; 18 : 303-15.

Detongnon J, Affokpon A. Étude du comportement agronomique de quelques variétés de niébé à usages multiples dans les régions du Nord-Est et Sud-Ouest du Bénin. In : Appui à la gestion de la recherche agricole nationale (AGRAN); faculté des sciences agronomiques (FSA); Institut national des recherches agricoles du Bénin (Inrab), (SNRA), ed. Actes de l'atelier scientifique 2001-2. Abomey-calavi (Bénin) : Inrab-cra-agonkanmey, 2001.

Grum M, Edwin, Gyasi A, Osei C, KranjacBerisavljevic G. Evaluation des meilleures pratiques pour la conservation des cultivars locaux. Notes techniques. Nairobi : International Plant Genetic Resources Institute (IPGRI), 2003.

Louette D, Smale M. Genetic diversity and maize seed management in a traditional Mexican community: implications for in situ conservation of maize. NRC Paper. Mexico : International Maize and Wheat Improvement Centre (CIMMYT), 1996.

Oyewole B, Carsky RJ, Schulz S. On-farm testing of Mucuna and cowpea double cropping with maize in the Guinea savanna of Nigeria. In : Carsky RJ, Keatinge JDH, Manyong VM, Eteka AC, eds. Cover crops for integrated natural resource management in West Africa. Proceedings of a Regional Workshop organised by IITA and CIEPCA, 26-29 October 1999, Cotonou, Benin. Ibadan : International Institute of Tropical Agriculture (IITA), 2000.

Sanginga N, Lyasse O, Singh BB. Phosphorus use efficiency and nitrogen balance of cowpea breeding lines in a low $\mathrm{P}$ soil of the derived savanna zone in West Africa. Plant Soil 2000 $220: 119-28$

Schulz S, Hussaini MA, Kling JG, Berner DK, Ikie FO. Evaluation of integrated Striga Hermonthica control technologies under farmer management. Exp Agric. Ibadan: International Institute of Tropical Agriculture (IITA), 2003.

Tall EK. Dynamique des cultes vodouns et du Christianisme au Sud-Bénin. Cah Sci Hum $1995 ; 31: 797-823$.

Zoundjihékpon J, Dansi A, Mignouna JHD, et al. Gestion des ressources génétiques des ignames africaines et conservation in situ. In : Institut d'économie rurale (IER); Bureau des ressources génétiques (BRG); (Solagral), ed. Gestion des ressources génétiques des plantes en Afrique des savanes. (Bamako (Mali) Paris ; Montpellier : IER ; BRG ; Solagral, 1997. 